Check for updates

Cite this: Phys. Chem. Chem. Phys., 2020, 22, 19162

Received 20th May 2020, Accepted 12th August 2020

DOI: $10.1039 / \mathrm{d} 0 \mathrm{cp} 02736 \mathrm{~g}$

rsc.li/pccp

\section{Electroresponsive structuring and friction of a non-halogenated ionic liquid in a polar solvent: effect of concentration $\dagger$}

\author{
Georgia A. Pilkington, (D)*a Anna Oleshkevych, ${ }^{a}$ Patricia Pedraz, ${ }^{a}$ \\ Seiya Watanabe, (D) $\ddagger^{a}$ Milad Radiom, (D) ${ }^{a}$ Akepati Bhaskar Reddy, ${ }^{b}$ Alexei Vorobiev, ${ }^{c}$ \\ Sergei Glavatskih (D) bd and Mark W. Rutland (D) ae
}

\begin{abstract}
Neutron reflectivity (NR) measurements have been employed to study the interfacial structuring and composition of electroresponsive boundary layers formed by an ionic liquid (IL) lubricant at an electrified gold interface when dispersed in a polar solvent. The results reveal that both the composition and extent of the IL boundary layers intricately depend on the bulk IL concentration and the applied surface potential. At the lowest concentration $(5 \% \mathrm{w} / \mathrm{w})$, a preferential adsorption of the IL cation at the gold electrode is observed, which hinders the ability to electro-induce changes in the boundary layers. In contrast, at higher IL bulk concentrations (10 and $20 \% \mathrm{w} / \mathrm{w}$ ), the NR results reveal a significantly larger concentration of the IL ions at the gold interface that exhibit significantly greater electroresponsivity, with clear changes in the layer composition and layer thickness observed for different potentials. In complementary atomic force microscopy (AFM) measurements on an electrified gold surface, such IL boundary layers are demonstrated to provide excellent friction reduction and electroactive friction (known as tribotronics). In agreement with the NR results obtained, clear concentration effects are also observed. Together such results provide valuable molecular insight into the electroactive structuring of ILs in solvent mixtures, as well as provide mechanistic understanding of their tribotronic behaviours.
\end{abstract}

\section{Introduction}

Ionic liquids (ILs) are attractive candidates for many energy applications, such as fuel and solar cells, supercapacitors and electrochemical devices, as well as in lubrication, where they have the potential to enhance energy efficiency and provide additional functionality. ${ }^{1}$ Due to their inherent ionic nature, the active control of the interfacial structures and resultant interfacial properties of ILs (e.g., friction) can be realised

\footnotetext{
${ }^{a}$ Division of Surface and Corrosion Science, School of Engineering Sciences in Chemistry, Biotechnology and Health, KTH Royal Institute of Technology, SE-100 44 Stockholm, Sweden. E-mail: georgiap@kth.se

${ }^{b}$ System and Component Design, Department of Machine Design, KTH Royal Institute of Technology, SE-100 44 Stockholm, Sweden ${ }^{c}$ Department of Physics and Astronomy, Division of Materials Physics, Uppsala University, Uppsala, Sweden

${ }^{d}$ Department of Electromechanical, Systems and Metal Engineering, Ghent University, B-9052 Ghent, Belgium

${ }^{e}$ Surfaces, Processes and Formulation, RISE Research Institutes of Sweden, SE-100 44 Stockholm, Sweden

$\dagger$ Electronic supplementary information (ESI) available. See DOI: 10.1039/ d0cp02736g

\# Current address: Tokyo University of Science, Department of Mechanical Engineering, 6-3-1 Niijuku, Katsushika-ku, Tokyo 125-8585, Japan.
}

through surface charge. ${ }^{2-8}$ Nonetheless, commercial application of ILs has been perturbed by a number of issues, including high production costs, generally poor miscibility in base fluids (e.g., mineral oil), as well as related to the common choice of fluorine-containing anions, which under tribological conditions (i.e., high temperatures and pressures) have been shown to form toxic and corrosive halogen halides. ${ }^{9,10}$

To address these issues, in recent years, there has been increasing demand for the synthesis of non-halogenated ILs with good miscibility in broad range of base fluids. In particular, ILs containing phosphorus- and boron-based ions have been identified as promising candidates for lubricant additives and have been shown to exhibit excellent electroresponsive and lubrication properties, both as neat lubricants and when dispersed at low concentrations in a base oil or greases. ${ }^{11-18}$ Yet, a mechanistic understanding of how the interfacial structures and composition of IL boundary layers in a base fluid can be modified and controlled by an electric field (i.e., realising tribotronics), and importantly, how such behaviours may be affected by the bulk IL concentration, is still lacking.

Only a limited number of studies have investigated the interfacial structures formed in IL solutions at charged interfaces. Using X-ray reflectivity (XRR), Mezger et al. showed the 
structuring of the halogenated IL [BMPy] $\left[\mathrm{NTF}_{2}\right]$ in the polar solvent propylene carbonate (PC) at a negatively charged sapphire surface to be strongly dependent on the IL concentration. ${ }^{19}$ With increasing PC concentration, the periodicity and correlation of the interfacial layers both increased and decreased, respectively, reflecting a reduction in the Coulomb interactions between the ions. For the electric field control of IL layers, reduced inter-ion interactions are likely to lead to an increased mobility of the ions and thus a greater electroresponsiveness. For example, the tribological electroresponsiveness of pure imidazolium ILs has been shown to be lower for those with longer alkyl chains and attributed to a greater cohesiveness between the (cat)ions, also reflected in their higher viscosity and lower conductivities. ${ }^{20}$ Meanwhile, recent macroscopic tribological studies on stainless steel have shown that variation of the adsorbed ionic species in halogenated imidazolium IL mixtures in PC with applied electric potential can also affect their wear behaviours. ${ }^{21,22}$

Using a surface force balance (SFB), Perkin et al. have studied the structuring of a fluorinated IL, [BMIM] $\left[\mathrm{NTF}_{2}\right]$, between two confining mica surfaces at low $(0.1 \mathrm{~mol} \%)$ and high (46 mol\%) concentrations in PC. ${ }^{23}$ At the low IL concentration, the periodicity of the confined layers correlated with that of the PC, suggesting the interfacial structuring at sufficiently low concentrations is largely determined by the solvent molecules, whilst at the higher concentration the periodicity was larger, and more comparable with the dimensions of the IL ions. In the same study, the number density of the IL ions and PC molecules was also predicted for different IL concentrations at an unconfined negatively charged surface. However, the magnitude or bias of the surface charge was not investigated.

Previously, we have studied the electroresponsive self-assembly of the IL trihexyl-(tetradecyl)phosphonium bis(mandelato)borate $\left[\mathrm{P}_{6,6,6,14}\right][\mathrm{BMB}]$ on an electrified gold surface, both as a pure IL and when dispersed in a polar solvent (acetone), using NR. ${ }^{24}$ By matching the scattering length density (SLD) of the bulk IL/solvent mixtures to the gold, the sensitivity of the reflected intensity to the potential-driven changes in the interfacial layer was dramatically improved compared to that afforded for pure IL systems. ${ }^{18,25}$ For different potentials, systematic changes in the reflectivity fringes were observed. By invoking a single layer model, systematic changes in the relative populations of the IL ions in the boundary layer with applied potential were revealed.

Here, we have chosen to focus on IL/solvent mixtures with PC, which is a widely regarded as a superior solvent for high permittivity electrolytes due to its high dielectric constant $\left(64\right.$ at $\left.25{ }^{\circ} \mathrm{C}\right)$. When deuterated, PC also has a high SLD $\left(5.66 \times 10^{-6} \AA^{-2}\right)$, which is essential for contrast matching the bulk solution with that of the gold electrode (SLD $\sim 4.56 \times$ $10^{-6} \AA^{-2}$ ). We present NR measurements for three different concentrations of the non-halogenated IL $\left[\mathrm{P}_{6,6,6,14}\right][\mathrm{BMB}]$ solution in PC at an electrified gold surface as a function of applied potential. In addition, nanotribological AFM and electrochemical impedance spectroscopy (EIS) measurements we performed for the lowest and highest IL concentrations on a gold electrodes to probe the tribotronic and charge responsivities of the IL mixtures. Together, the results provide clear evidence that both structural and compositional changes in the boundary layers with applied potential are responsible for the measured electroresponsive friction (i.e., tribotronic properties) of the IL layers and suggest that the nature and magnitudes of these electro-induced changes are strongly dependent on the bulk IL concentration.

\section{Materials and methods}

\subsection{Materials and solution preparation}

The IL trihexyl(tetradecyl)phosphonium-bis(mandelato)borate, $\left[\mathrm{P}_{6,6,6,14}\right][\mathrm{BMB}](c f$. Fig. 1 ) was synthesised at Luleå University of Technology. ${ }^{11}$ This IL was chosen due to its excellent lubrication and electro-responsive properties. ${ }^{6,10,11,17,26}$ Prior to all measurements the pure $\left[\mathrm{P}_{6,6,6,14}\right][\mathrm{BMB}]$ was baked under vacuum at $60^{\circ} \mathrm{C}$ for approximately $72 \mathrm{~h}$ to remove traces of solvents and water. The solvents, PC (anhydrous, 99.7\%, Sigma Aldrich) and 1,2-propylene- $\mathrm{d}_{6}$ carbonate (d-PC; 99.5 atom \% D, Qmx Laboratories Ltd), were used as received. All IL solutions were prepared by weighing the IL and adding the appropriate volume of the solvents. The concentrations of the solutions are presented in terms of weight percentage $(\% \mathrm{w} / \mathrm{w})$. The IL concentrations of 5,10 and $20 \% \mathrm{w} / \mathrm{w}$ in PC, correspond to 0.67 , 1.4 and $3.1 \mathrm{~mol} \%$ of the IL, respectively.

For NR measurements, the bulk SLD of all solutions was contrast matched to (or as closely as possible to) that of the gold $\left(4.65 \times 10^{-6} \AA^{-2}\right)$. The solutions were sonicated for at least 10 minutes until homogeneous (by eye) and used immediately, or stored under nitrogen. ATR-IR measurements (FT/IR - 4600 and ATR PRO ONE Single-reflection ATR accessory, Jasco, Japan), were performed on the IL solutions to obtain molecular vibration information and to determine if any water was present in the IL solutions. A diamond crystal was used for the ATR prism. A droplet of IL solution was placed on the top of the ATR prism. The spectral resolution was fixed to $4 \mathrm{~cm}^{-1}$ and number of accumulations was 64 , corresponding to a 1 min sampling time. The IR spectra for the 10 and $20 \% \mathrm{w} / \mathrm{w}$ solutions used for

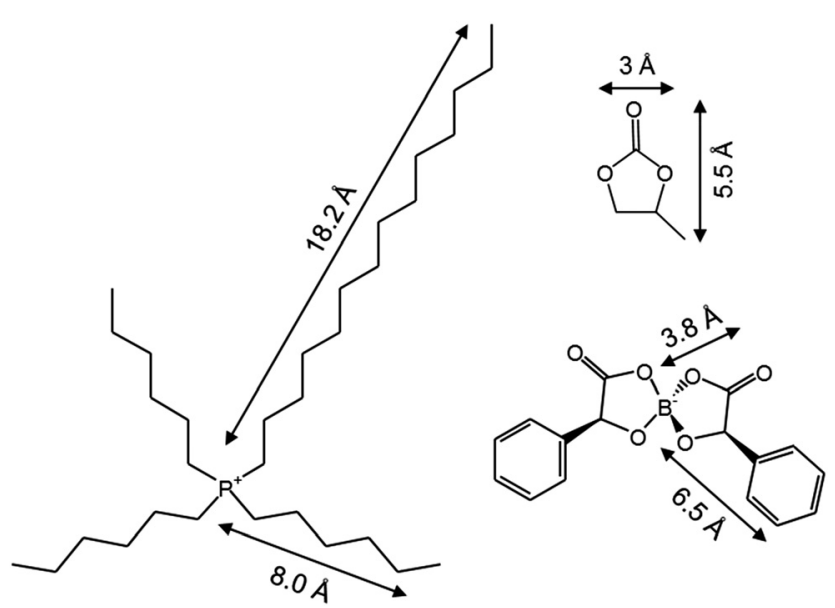

Fig. 1 Molecular structures and dimensions of trihexyltetradecylphosphonium bis(mandelato)borate, $\left[\mathrm{P}_{6,6,6,14}\right][\mathrm{BMB}]$, and the solvent, $\mathrm{PC}$. 
the NR measurements are provided in the ESI $\dagger$ ( $c f$. S5) and did not exhibit any distinguishable water peaks.

\subsection{Preparation of gold surfaces}

Thin ( $\sim 170 \AA$ ̊), amorphous gold films were prepared by electron beam evaporation (Auto 306, Edwards High Vacuum International, Wilmington, MA) atop of different silicon substrates and used as working electrodes. For the NR measurements, the films were deposited on $10 \mathrm{~mm}$ thick $(50 \times 50 \mathrm{~mm})$ polished silicon (100) blocks (Sil'tronix Silicon Technologies, France). For the AFM and electrochemical impedance spectroscopy (EIS) measurements, the films were coated thin $(0.5 \mathrm{~mm})$ polished silicon (100) wafer chips (University Wafer, Boston, MA, US). To ensure good adhesion between the $\mathrm{Si}$ and $\mathrm{Au}$, the Si surfaces were pre-coated with a titanium $(\sim 30 \AA)$ adhesion layer. Prior to deposition, the Si surfaces were cleaned in Piranha solution, rinsed with Milli-Q water and dried with filtered $\mathrm{N}_{2}$. Before all measurements, the coated surfaces were rinsed with filtered ethanol, blow dried with nitrogen and exposed to UV/ozone for 10 minutes. Prior to all solution NR measurements, a short length of insulated copper wire was adhered to one corner of each gold surface using a conductive epoxy (CW400, Chemtronics), which was cured in an oven at $120{ }^{\circ} \mathrm{C}$ for $20-30$ minutes before repeating the same cleaning procedure.

\subsection{Neutron reflectivity measurements and analysis}

The NR measurements presented here were performed on the SuperADAM reflectometer at the Institut Laue-Langevin (ILL), Grenoble, France using a custom-made electrochemical NR cell. ${ }^{24}$ Briefly, the neutron specular reflectivity $R$ (normalised by the incident intensity) was measured as a function of the momentum transfer vector $Q_{\mathrm{z}}=4 \pi \sin \theta / \lambda$ directed perpendicular to the sample surface, where $\theta$ is the angle of incidence and $\lambda$ is the wavelength, which was fixed at $5.2 \AA$. Before exposing the gold films to the IL solutions, the reflectivity from the coated blocks was first measured in air to characterise the thickness and roughness of the $\mathrm{Si}, \mathrm{SiO}_{2}$, $\mathrm{Ti}$ and $\mathrm{Au}$ layers using known values for the SLD of each layer ( $c f$. ESI; $\dagger$ Fig. S1). For NR solution measurements, the neutron beam was directed through the Si block and reflected from the gold-solution interface, with the reflected beam again traversing the block and exiting to the detector. To reduce background scattering, cadmium slits were positioned before and behind the liquid cell.

The scattering data were reduced using the program SARED provided by SuperADAM, ILL. The data were corrected using standard background subtraction and detector efficiency calibration followed by normalisation of the reflected intensity to the direct incident beam and an over illumination correction. The error bars for all the reflectivity data presented are calculated based on a Poisson distribution, whereby the count error is given by the square root (or standard deviation) of the number of counts measured at each position in $Q_{\mathrm{z}}$. These are then propagated using a standard error propagation with the errors associated with the monitor and the direct beam, that are also calculated in the same way, in order to obtain the total error in the reflectivity.
Table 1 Neutron SLDs and molecular volumes $\left(V_{M}\right)$ of $\left[P_{6,6,6,14}\right][B M B]$ and its constituent ions

\begin{tabular}{lcr}
\hline Species & $\operatorname{SLD}\left(10^{-6} \AA^{-2}\right)$ & $V_{\mathrm{M}}\left(\AA^{3}\right)$ \\
\hline$\left[\mathrm{P}_{6,6,6,14}\right][\mathrm{BMB}]$ & 0.50 & 1291 \\
{$\left[\mathrm{P}_{6,6,6,14}\right]^{+}$} & -0.40 & 912 \\
{$[\mathrm{BMB}]^{-}$} & 2.68 & 378
\end{tabular}

The SLD profiles were obtained from best-fits to the NR data performed using the software package GenX and a logarithmic figure of merit (FOM). ${ }^{27}$ All parameters related to the substrate, i.e. the $\mathrm{Au}, \mathrm{Ti}$ and $\mathrm{SiO}_{2}$ thicknesses and SLDs, as well as the $\mathrm{Au}, \mathrm{Ti}^{2} \mathrm{SiO}_{2}$ and Si roughnesses, were obtained from the reflectivity of the blocks measured in air and fixed for all subsequent conditions ( $c$. ESI; $\dagger$ Fig. S1 and Table S1). The SLD of the bulk solution was also kept constant for the different potential conditions studied. Initial fits were performed for a one-layer model and subsequent layers added if the FOM value decreased. The simulations were performed independently and no pre-defined structures were assumed. For all the fitted datasets, the thickness and SLD of the interfacial layers, as well as the roughnesses of the gold/IL and IL/bulk interfaces were allowed to vary. The gold roughness was limited to a minimum value of that measured in air. The layer SLDs were constrained to be within the range of the component species ( $c f$. Table 1). The estimated SLD of the bulk IL was calculated from its mass density $\left(1.022 \mathrm{~g} \mathrm{~cm}^{-3}\right)$, whilst the SLDs of the ions were estimated from their calculated molar volumes ( $c f$. Table 1).

Details regarding the electrochemical NR cell design have been reported previously in a related study of IL-solvent mixtures. ${ }^{24}$ However, briefly, before assembling the cell, the conductive glass (coated with a fluorine-doped tin oxide, FTO; NSG TEC A7, Pilkington, UK) used as counter electrode, as well as all the other cell components, were sonicated in $2 \%$ Hellmanex for 30 minutes, before being rinsed thoroughly with Milli-Q water and absolute ethanol, and dried with filtered $\mathrm{N}_{2}$. For the measurements presented here, a PTFE gasket of $0.5 \mathrm{~mm}$ thickness was used to separate the working (gold films) and counter electrodes. In order to minimise ambient water contamination, the cell was assembled inside a polyethylene glove bag under a dry, nitrogen atmosphere (R.H. $<10 \%$ ). The IL solutions were injected into the bottom port of the cell through PTFE tubing using a glass Luer syringe. Once mounted on the beamline, electrical potentials were applied across the NR cell using a potentiostat (Metrohm Autolab, PGSTAT204). Before applying a potential, the open circuit potential (OCP) was measured across the NR cell. Depending on the concentration of IL, the OCP value varied between -0.05 and $-0.1 \mathrm{~V}$. During all electrochemical NR measurements, the applied potential and current across the cell were continuously recorded and monitored. The electric potentials were applied in the same order as presented. For each potential, the potential was applied for 30 minutes prior to the NR measurements to allow for the cell current to stabilise ( $c f$. ESI; $†$ Fig. S6) and an equilibrium state to be reached.

\subsection{Tribotronic friction force measurements}

Electrochemical friction force measurements were performed using a JPK Nanowizard AFM equipped with a JPK ECCell ${ }^{\mathrm{TM}}$ 
electrochemical cell. Friction was measured between a sharp $\mathrm{Si}$ AFM tip (radius $R=7 \mathrm{~nm}$; AC204TS, Olympus) with a spring constant of $1.5 \mathrm{~N} \mathrm{~m}^{-1}$, measured by the thermal tune method, and a gold coated silicon wafer which acted as a working electrode. The potential was applied using a PGU BI-1000 potentiostat (Ingenieurbüro Peter Schrems (IPS), Germany). Pt wires were used as counter (CE) and reference (RE) electrodes. Ferrocene was used as an internal standard to determine the potential of the Pt pseudo-reference electrode $v s . E_{1 / 2}\left(\mathrm{Fc}^{+} / \mathrm{Fc}\right)$, as described in Section 2.5.

Prior to the measurements, the electrochemical cell was dissembled and cell parts were thoroughly cleaned using acetone, isopropanol (IPA) and absolute ethanol, before being dried with filtered nitrogen. The AFM tip and gold surface were also rinsed with absolute ethanol and irradiated in an ultraviolet (UV) ozone cleaner (PCE-44-LD; MTI Corporation, US) for 10 minutes. Friction measurements were performed over a scan size of $500 \mathrm{~nm}$ and at a scan rate of 1,6 and $12 \mu \mathrm{m} \mathrm{s}^{-1}$. The lateral deflections, measured as changes in the lateral voltage signal $\Delta V$, were averaged over 10 trace and retrace cycles at each normal load. The averaged lateral signals were converted to friction force using the following equation:

$$
F_{\mathrm{f}}=\frac{\Delta V k_{\phi}}{2 h_{\mathrm{eff}} \delta},
$$

where $k_{\phi}$ is the torsional spring constant, $h_{\text {eff }}$ is the effective height of the AFM tip (length plus half the thickness of the cantilever) and $\delta$ is the lateral reflection sensitivity. ${ }^{28,29} k_{\phi}$ was calculated using the Hybrid method developed by ÁlvarezAsencio et $a l .{ }^{30}$ The lateral sensitivity of the AFM photodiode was calibrated in PC and corrected for the refractive index $(\mathrm{RI}=1.424)$ of the IL solution, ${ }^{31}$ which was determined using an Abbe refractometer. The friction coefficient $\mu$ was obtained for each data set, where $\mu$ is extracted from the gradient of the friction force versus the normal load $F_{\mathrm{N}}$ in the linear region. To minimise water absorption, all AFM measurements were performed under an Argon atmosphere. The OCP of the solution was $-120 \mathrm{mV}$. All potentials are given as the absolute potential and were applied for 30 minutes prior to each set of friction measurements. Friction measurements were also performed in a pure solvent, PC. The average friction coefficient for PC (without potential) was $\sim 0.14$ at $6 \mu \mathrm{m} \mathrm{s}^{-1}$ (cf. Fig. 5c).

\subsection{Cyclic voltammetry and electrochemical impedance spectroscopy}

CV measurements were performed across the NR and AFM electrochemical cells to establish that no faradaic events occurred within the potential window studied ( $c f$. ESI, $\dagger$ Fig. S4). ${ }^{24}$ During the CVs, the potential was cycled at a scan rate of $10 \mathrm{mV} \mathrm{s}^{-1}$ starting at $0 \mathrm{~V}$ in the anodic scan direction. In addition, cyclic voltammetry measurements were performed across freshly prepared IL solutions with $100 \mathrm{mM}$ ferrocene to obtain the half wave potential $\left(E_{1 / 2}\right)$ of the ferrocene/ferrocenium $\left(\mathrm{Fc}^{+} / \mathrm{Fc}\right)$ redox pair. This internal standard allows for comparison between different electrochemical cells or setups.
A TSC Surface measuring cell (rhd instruments GmbH \& Co. KG) was used for the EIS measurements. A gold-coated silicon piece (prepared in the same way as for NR and AFM surfaces, see Section 2.2) was used as working electrode (WE) and was contacted from the topside, outside of the liquid area. The liquid area was defined by a FFKM o-ring, with an inner diameter of $6 \mathrm{~mm}$. A glassy carbon electrode pressed in PEEK (diameter of active electrode surface equal to $6 \mathrm{~mm}$ ) was used as counter electrode. As pseudo reference electrode, a silver wire was used. The sample temperature was actively controlled by a Microcell HC setup (rhd instruments GmbH \& Co. KG) and set to $20{ }^{\circ} \mathrm{C}$ for each measurement. Before each experiment, the cell was thoroughly cleaned with ethanol and acetone, dried at $60{ }^{\circ} \mathrm{C}$ for $2 \mathrm{~h}$ in a vacuum oven, and transferred into a glovebox where it was assembled and filled, ensuring minimum exposure to ambient water.

As a measuring device, a PGSTAT302N potentiostat/galvanostat (Methohm Autolab) equipped with a FRA32 module was used. After determining the OCP, the WE potential was set $0 \mathrm{~V}$ $v s$. RE. Impedance spectra for frequencies ranging from $10 \mathrm{mHz}$ to $100 \mathrm{kHz}$ were recorded applying an ac voltage amplitude of $10 \mathrm{mV}$ (rms) and using automated current ranging. The stabilisation waiting time in between each potential step $( \pm 0.25 \mathrm{~V})$ was $1800 \mathrm{~s}$. Anodic and cathodic scan directions were recorded using different gold working electrodes and the cell cleaned in between. After finishing the impedance measurements in each scan direction, ferrocene was dissolved in the solution (inside the glove box) to perform a CV to determine the potential of the silver pseudo-reference electrode vs. $E_{1 / 2}\left(\mathrm{Fc}^{+} / \mathrm{Fc}\right)$. For data evaluation, the RelaxIS 3 software (rhd instruments $\mathrm{GmbH}$ \& Co. KG) was used.

\section{Results and discussion}

\subsection{Neutron reflectivity}

3.1.1 5\% $\left[\mathbf{P}_{6,6,6,14}\right][\mathrm{BMB}]$ in PC. Fig. 2a shows the reflectivities for a $5 \% \mathrm{w} / \mathrm{w}$ solution of $\left[\mathrm{P}_{6,6,6,14}\right][\mathrm{BMB}]$ in $\mathrm{PC}$ at a gold electrode interface for three potential conditions: $0 \mathrm{~V},-1.5 \mathrm{~V}$ and $+0.25 \mathrm{~V}$. For clarity the curves have been offset from each other in the $y$-axis. Due to the strong contrast between the interfacial IL region and the gold electrode the reflectivity curves show pronounced Kiessig fringes, that would not be present in the absence of an interfacial boundary layer (dashed line). ${ }^{24}$ To compare the relative changes in the reflectivity curves with respect to $0 \mathrm{~V}$, corresponding asymmetry plots are shown in the Fig. 2a inset. For both the negative and positive potential, the asymmetry curves show clear, but different, systematic deviations, signifying an electroresponsivity for both biases. However, the magnitude of the deviations in the asymmetry signal for the positive potential were notably larger. Such deviations at different applied potentials were not observed in the reflectivity profiles for the pure solvent, PC ( $c f$. ESI, $\dagger$ Fig. S2).

For all potentials, a one-layer slab model was sufficient to describe the data well and the addition of multiple layers did not found to improve the quality of the fits. Previous AFM 
(a)

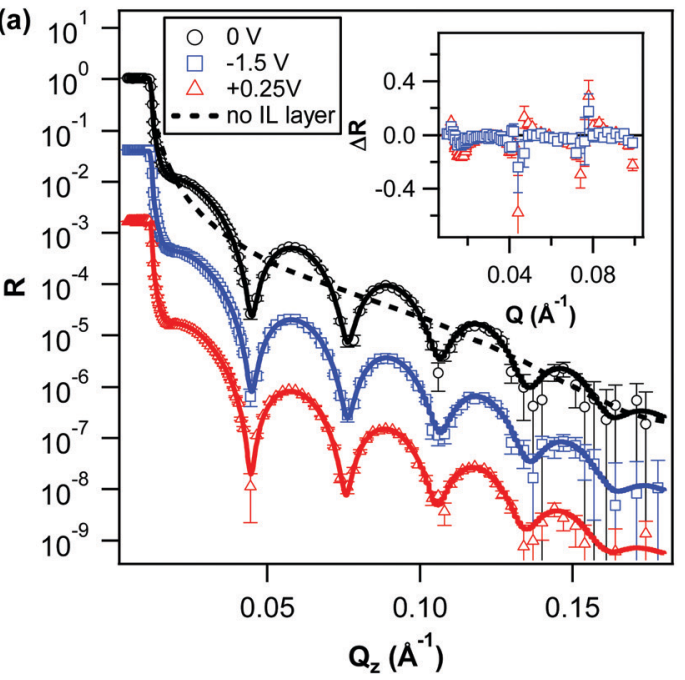

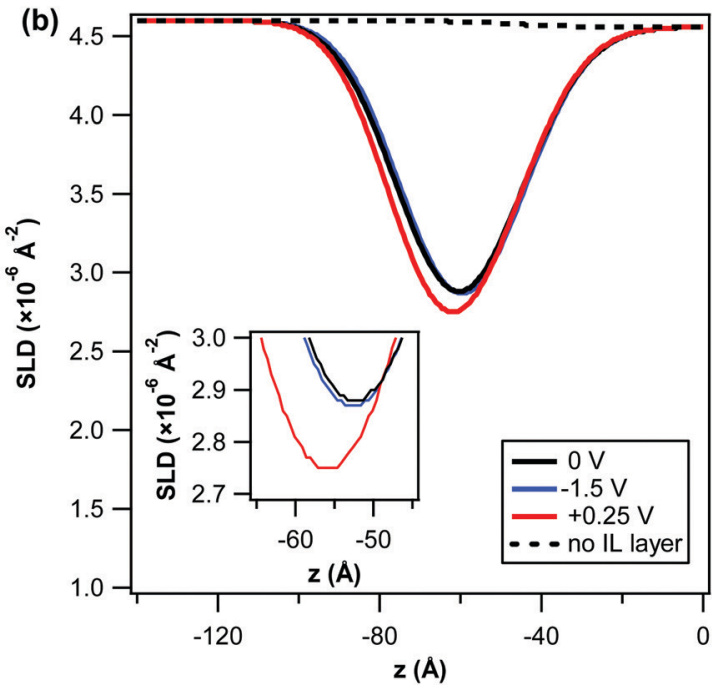

Fig. 2 (a) Experimental neutron reflectivity, $R$, of $5 \% \mathrm{w} / \mathrm{w}\left[\mathrm{P}_{6,6,6,14}\right][\mathrm{BMB}]$ in $\mathrm{PC}$ for different applied potentials $(0,-1.5$, and $+0.25 \mathrm{~V})$ plotted as a function of the momentum transfer vector $Q_{z}$ at different applied potentials. The symbols show experimental data whilst the solid lines represent best-fits to the data for each potential. The error bars represent the statistical error (standard deviation) in the experimental reflectivity data. For clarity, the curves have been offset in the $y$-axis. For comparison, the dashed line shows the predicted reflectivity for the same surface and solution without an interfacial IL layer. The inset shows asymmetry plots $\Delta R=\left[R^{\vee}\left(Q_{\mathrm{z}}\right)-R^{0}\left(Q_{\mathrm{z}}\right)\right] /\left[R^{\vee}\left(Q_{\mathrm{z}}\right)+R^{0}\left(Q_{\mathrm{z}}\right)\right]$, obtained from the experimental data, highlighting the changes in the non-zero potential reflectivities $\left(R^{\vee}\right)$ with respect to $0 \vee\left(R^{0}\right)$. (b) Corresponding model SLD profiles obtained from the best-fits to the reflectivity profiles as a function of distance $(z)$, where $z=0$ represents the gold-solution interface. The inset shows an expanded area of the main figure around the SLD minima corresponding to the IL interfacial region.

force-distance measurements have also revealed a monotonic squeeze-out force in the same pure IL, suggesting the bulky and highly delocalised IL ions do not easily undergo interfacial stratification, which has hitherto been considered a characteristic of ionic liquids. ${ }^{32}$ Corresponding SLD profiles obtained from best-fits to the data are shown in Fig. $2 b$, whilst the specific parameters obtained from the fits (i.e. roughnesses, layer thickness and SLD) can be found in the ESI $\dagger$ ( $c f$. Table S2). Overall, the SLD profiles reveal a broad interfacial region, with high roughness and a relatively low SLD. With applied potential, the SLD profiles show a relatively weak response in the surface structure to the negative potential $(-1.5 \mathrm{~V})$, whereby the thickness and average SLD of the layer are only slightly reduced, indicating a more compact structuring in the interfacial region. In contrast, at the positive potential $(+0.25 \mathrm{~V})$, a more pronounced broadening of the boundary layer into the bulk solution and change in SLD is observed. In the best-fit SLD model for this layer the SLD is also somewhat higher, suggesting a more mixed layer of higher anion concentration ( $c f$. ESI; $\dagger$ Table S2). However, this SLD increase is not reflected in the SLD profile, due to an apparent simultaneous increase in the layer thickness and decrease in interfacial layer/bulk roughness. It is possible therefore that at this low IL concentration, a "sensitivity limit" is met, probably due to the highly diffusive nature of the interfacial region (i.e., high solvent content) at this (bulk) IL concentration.

The weak response to a negative bias, and overall relatively low SLD of the IL boundary region (equivalent to $\sim 62 \%$ of the pure IL, assuming the same ratio of the hydrogenated and $d$-PC as in the bulk; $c f$. ESI, $\dagger$ Table S2), suggest a higher concentration of the cation to be already present at the gold interface at $0 \mathrm{~V}$. Together these observations point towards a preferential adsorption of the IL cation at the gold interface. Such findings are consistent with previous results of the same ionic liquid on gold in another polar solvent. ${ }^{24}$ Moreover, a specific interaction between the same cation and a neutral gold electrode has also been predicted before for the pure IL by molecular dynamic simulations. ${ }^{26}$ In another recent NR study, the absorbed layers of an IL containing the same cation also suggest a predominance of cations at a negatively charged silica interface in IL-oil mixtures. ${ }^{33}$ Here, at this low bulk IL concentration, this specific interaction with the gold electrode therefore is found to dominate the IL boundary layer structure and limit its ability to responds to an external electric field.

3.1.2 10\% $\left[\mathbf{P}_{\mathbf{6}, 6,6,14}\right][\mathrm{BMB}]$ in PC. Fig. 3a shows the reflectivities for a $10 \% \mathrm{w} / \mathrm{w}$ solution of $\left[\mathrm{P}_{6,6,6,14}\right][\mathrm{BMB}]$ in $\mathrm{PC}$ at a gold interface at the three same potentials $(0,-1.5 \mathrm{~V}$ and $+0.25 \mathrm{~V})$ as those presented for $5 \% \mathrm{w} / \mathrm{w}$ that were applied in the same order. Compared to the lower IL concentration, the corresponding asymmetry curves in the inset of Fig. 3a reveal more pronounced changes for both potential biases with respect to $0 \mathrm{~V}$. In particular, clear shifts in the Kiessig fringes from the gold interface suggest notable changes in the apparent thicknesses of the interfacial layers. Compared to $5 \% \mathrm{w} / \mathrm{w}$, the magnitudes of the asymmetry signals for both biases are also more comparable, indicating a larger change in the SLD of the boundary region was achieved at the applied negative potential.

The corresponding SLD profiles to the best-fits to the reflectivity curves are shown in Fig. 3b. For all potential conditions, the reflectivity profiles were best described by a one-layer model. Compared to $5 \% \mathrm{w} / \mathrm{w}$, the minima in the SLD profiles (corresponding to the interfacial region) were overall higher 

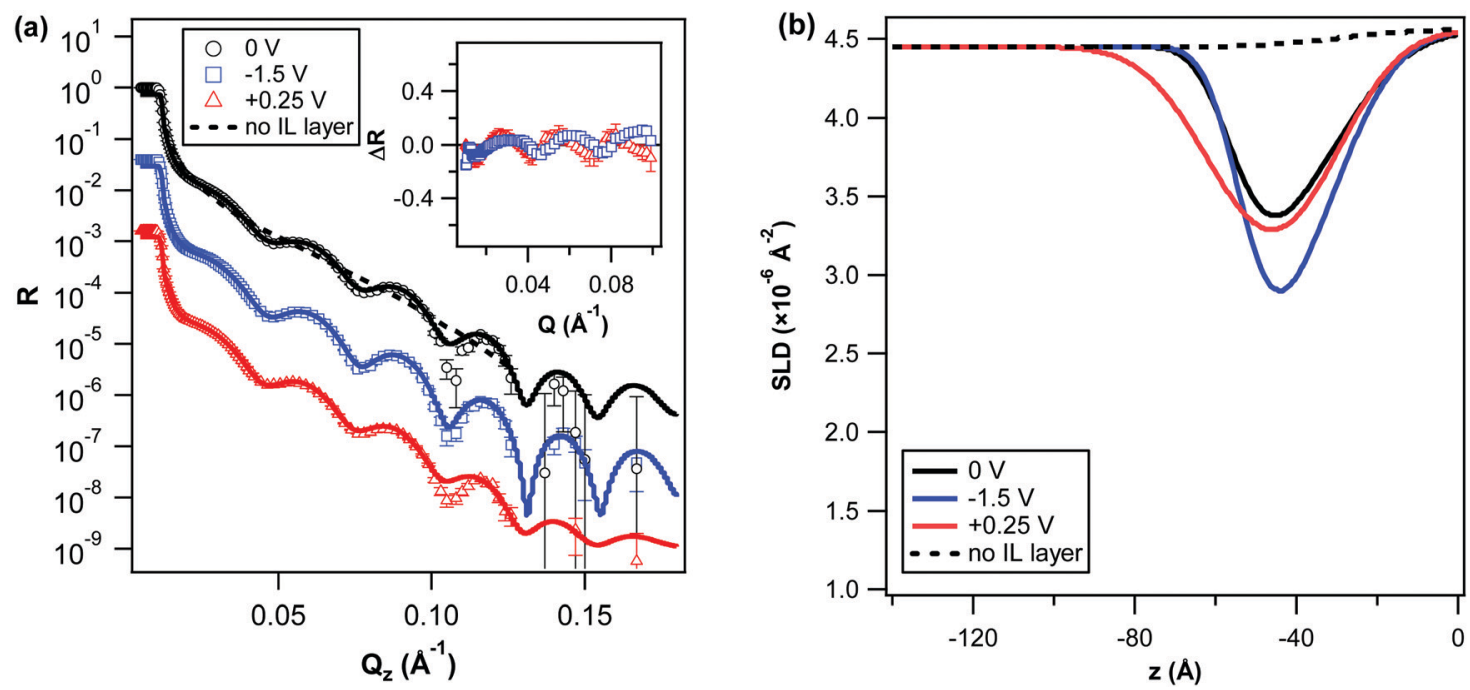

Fig. 3 (a) Experimental neutron reflectivity, $R$, of $10 \% \mathrm{w} / \mathrm{w}\left[\mathrm{P}_{6,6,6,14}\right][\mathrm{BMB}]$ in $\mathrm{PC}$ for different applied potentials $(0,-1.5$, and $+0.25 \mathrm{~V})$ plotted as a function of the momentum transfer vector $Q_{z}$ at different applied potentials. The symbols show experimental data whilst the solid lines represent best-fits to the data for each potential. The error bars represent the statistical error (standard deviation) in the experimental reflectivity data. For clarity, the curves have been offset in the $y$-axis. For comparison, the dashed line shows the predicted reflectivity for the same surface and solution without an interfacial IL layer. The inset shows asymmetry plots $\Delta R=\left[R^{\vee}\left(Q_{\mathrm{z}}\right)-R^{0}\left(Q_{\mathrm{z}}\right)\right] /\left[R^{\vee}\left(Q_{\mathrm{z}}\right)+R^{0}\left(Q_{\mathrm{z}}\right)\right]$, obtained from the experimental data, highlighting the changes in the non-zero potential reflectivities $\left(R^{\vee}\right)$ with respect to $0 \vee\left(R^{0}\right)$. (b) Corresponding model SLD profiles obtained from the best-fits to the reflectivity profiles as a function of distance $(z)$, where $z=0$ represents the gold-solution interface.

in SLD. At $0 \mathrm{~V}$, the SLD of the interfacial layer ( $c f$. ESI; $\dagger$ Table S3) is equivalent $\sim 45 \%$ of the bulk IL. This higher SLD suggests the absence of a preferential adsorption of the cation at the gold interface, as observed for $5 \% \mathrm{w} / \mathrm{w}$. Furthermore, the more narrow minima for all of the potentials also points towards a less diffuse (lower solvent content) structuring of the IL ions at the interface.

At $-1.5 \mathrm{~V}$ the deepening of the interfacial layer minimum in the SLD profile reveals a clear and significant change in the IL structuring in response to the negative bias and signifies a strong enhancement in the relative population of the IL cations at the interface. The more subtle reduction in the thickness and interfacial roughnesses of this layer ( $c f$. ESI; $†$ Table S3) further suggests a higher density of cations leads to a more compact structuring, consistent with the observed (weak) SLD change at $-1.5 \mathrm{~V}$ for $5 \% \mathrm{w} / \mathrm{w}$. Similar to that observed for $5 \% \mathrm{w} / \mathrm{w}$, the SLD profile for $+0.25 \mathrm{~V}$ also reveals the extent of the interfacial region to increase. Although, this effect is notably more pronounced in the case of $10 \% \mathrm{w} / \mathrm{w}$. Coupled with an increase in the SLD of the IL interfacial region and increased roughness with the bulk solution ( $c f$. ESI; $\dagger$ Table S3) the SLD profile for $+0.25 \mathrm{~V}$ and thus suggests a more disordered and mixed interfacial region, that possibly contains a higher concentration of anions. However, the higher SLD of the layer could also be attributable to a higher d-PC/PC content.

3.1.3 20\% PBMB in PC. The reflectivities for a $20 \% \mathrm{w} / \mathrm{w}$ solution of $\left[\mathrm{P}_{6,6,6,14}\right][\mathrm{BMB}]$ in $\mathrm{PC}$ at a gold electrode interface at different applied potentials are shown in Fig. 4a. In comparison to the previous lower two concentrations (5 and $10 \% \mathrm{w} / \mathrm{w}$ ), significantly larger changes were observed in the reflectivity profiles, as shown in the asymmetry plots in the inset of Fig. 4a.
Consistent with the other concentrations, one-layer slab models similarly best described the reflectivity profiles. Compared to the lower IL concentrations, the SLD of the interfacial region was significantly lower, as revealed by a deeper minimum in the SLD profiles. The extent of the IL layer was also markedly larger for all potentials compared to that at $10 \% \mathrm{w} / \mathrm{w}$, suggesting a thicker more densely packed interfacial region of IL ions.

Comparable to $10 \% \mathrm{w} / \mathrm{w}$, the SLD profile for $-1.5 \mathrm{~V}$ shows a clear decrease in the SLD of the interfacial region. However, in the case of $20 \% \mathrm{w} / \mathrm{w}$ the SLD of the minimum is notably lower suggesting a significantly higher density of the IL cations at the gold interface. Upon reversing the potential bias, the SLD profile for $+0.25 \mathrm{~V}$ also shows this process to be reversible, whereby the SLD of the region increases beyond that at $0 \mathrm{~V}$ and its extent into the bulk increases beyond that observed for lower IL concentrations. Together these changes in the SLD profile again infer there is a large increase in the population of anions at the interface upon application of a positive bias and that this in turn leads to a significantly less ordered and diffuse structuring.

\subsection{Tribotronic friction forces}

AFM has been shown to be a valuable tool for investigating the nanotribological properties of ILs and their mixtures at electrode interfaces. ${ }^{3,34-36}$ Here, nanoscale tribotronic friction measurements were conducted between a sharp Si tip and an electrified gold thin film in 5 and $20 \% \mathrm{w} / \mathrm{w}$ solutions of $\left[\mathrm{P}_{6,6,6,14}\right][\mathrm{BMB}]$ in $\mathrm{PC}(c f$. Fig. 5$)$ at several applied potentials. The lateral friction force was measured during loading and unloading between 0 to $200 \mathrm{nN}$. The potentials were applied by alternating bias and in order of increasing magnitude. For both IL concentrations studied, the friction coefficient also did not 

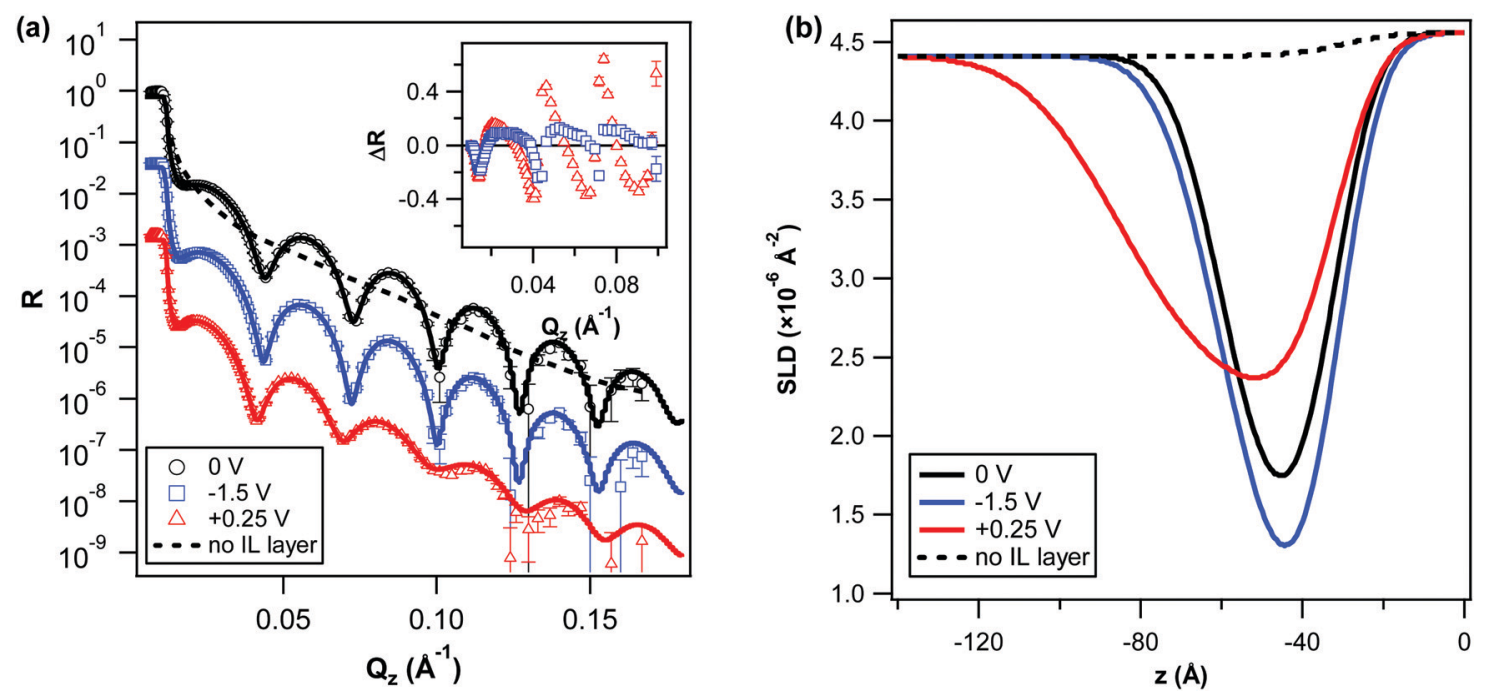

Fig. 4 (a) Experimental neutron reflectivity, $R$, of $20 \% \mathrm{w} / \mathrm{w}\left[\mathrm{P}_{6,6,6,14}\right][\mathrm{BMB}]$ in $\mathrm{PC}$ for different applied potentials $(0,-1.5$, and $+0.25 \mathrm{~V})$ plotted as a function of the momentum transfer vector $Q_{z}$ at different applied potentials. The symbols show experimental data whilst the solid lines represent best-fits to the data for each potential. The error bars represent the statistical error (standard deviation) in the experimental reflectivity data. For clarity, the curves have been offset in the $y$-axis. For comparison, the dashed line shows the predicted reflectivity for the same surface and solution without an interfacial IL layer. The inset shows asymmetry plots $\Delta R=\left[R^{\vee}\left(Q_{\mathrm{z}}\right)-R^{0}\left(Q_{\mathrm{z}}\right)\right] /\left[R^{\vee}\left(Q_{\mathrm{z}}\right)+R^{0}\left(Q_{\mathrm{z}}\right)\right]$, obtained from the experimental data, highlighting the changes in the non-zero potential reflectivities $\left(R^{\vee}\right)$ with respect to $0 \vee\left(R^{0}\right)$. (b) Corresponding model SLD profiles obtained from the best-fits to the reflectivity profiles as a function of distance $(z)$, where $z=0$ represents the gold-solution interface.

vary significantly for the different velocities measured $(1,6$ and $12 \mu \mathrm{m} \mathrm{s}^{-1}$; cf. Fig. $5 \mathrm{a}$ and b insets), as predicted by Amonton's law, and shown little evidence of hysteresis between loading and unloading cycles. For clarity, the friction forces for the just three potentials (OCP, $-1 \mathrm{~V}$ and $+0.25 \mathrm{~V}$ ) are shown as a function of applied load are shown for the intermediate velocity $\left(6 \mu \mathrm{m} \mathrm{s}^{-1}\right)$. Meanwhile, the friction coefficients measured for all potentials studied are shown in Fig. $5 \mathrm{c}$.

For the $5 \% \mathrm{w} / \mathrm{w}$ IL solution, the friction coefficient was reduced by $29 \%$ compared to that of PC at open circuit potential (OCP; $c f$. Fig. 5a). This reduction in nano-friction is comparable for that reported for another phosphonium-based IL with the same cation in hexadecane at a similar concentration $(1 \mathrm{~mol} \%)$ on silicon. ${ }^{12}$ With applied potential, the magnitude of the friction was found to vary, whereby a positive bias led to larger friction and a negative led to a reduced friction. At $20 \% \mathrm{w} / \mathrm{w}$, similar trends with applied potential were observed in the measured friction ( $c f$. Fig. $5 b$ ). From the NR SLD profiles at $20 \% \mathrm{w} / \mathrm{w}$ (Fig. $4 \mathrm{~b}$ ), it can be postulated that the lubricous behaviour of the IL at the negative potentials is due to a densely packed, cation rich layer, whilst higher friction towards positive potentials is found to correlate with a more
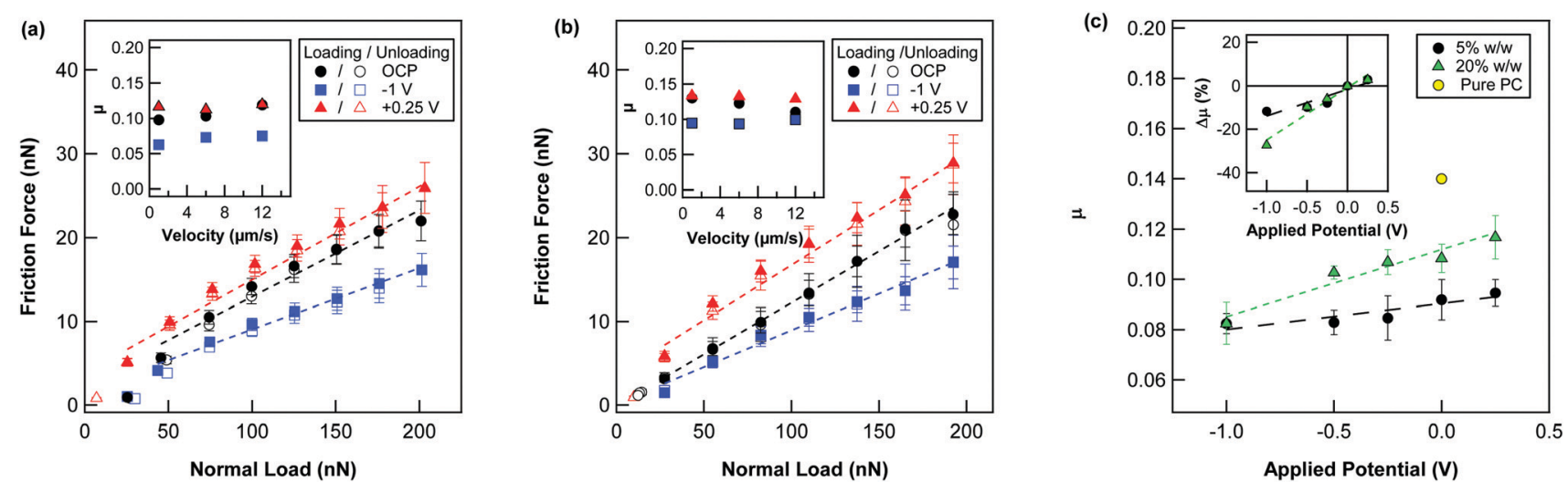

Fig. 5 Friction force as a function of normal load in a solution of (a) 5 and (b) $20 \% \mathrm{w} / \mathrm{w}\left[\mathrm{P}_{6,6,6,14}\right][\mathrm{BMB}]$ in PC on a gold electrode surfaces at three applied potentials (OCP, -1 and $+0.25 \mathrm{~V}$ ) during loading (closed symbols) and unloading (open symbols) measured at a velocity of $6 \mu \mathrm{m} \mathrm{s}{ }^{-1}$. The lines represent linear fits to loading datasets used obtain corresponding friction coefficients. The inset shows the friction coefficients for three different velocities $\left(1,6\right.$ and $\left.12 \mu \mathrm{m} \mathrm{s}^{-1}\right)$. (c) Friction coefficient $\mu$ as a function of applied potential during loading and unloading at a velocity of $6 \mu \mathrm{m} \mathrm{s}{ }^{-1}$. The inset shows the same data plotted as a percentage of the friction coefficient at $\mathrm{OCP}(\Delta \mu)$ for unloading and loading, respectively. The friction coefficient of the pure solvent on gold (without potential) at $6 \mu \mathrm{m} \mathrm{s}^{-1}$ is also shown for comparison. 
diffuse, extended anion-rich boundary layer. Slightly higher friction coefficients towards slower velocities at the positive potential also suggest that the anions are not strongly bound to the surface and are thus more easily sheared away from the surface at higher velocities ( $c f$. Fig. $5 b$ ).

For both concentrations, the magnitude of the friction coefficient varied linearly with applied potential ( $c f$. Fig. 5c). However, a larger tribotronic responsive was measured for the higher bulk IL concentration. This result is consistent with the NR SLD profiles for 5 and $20 \% \mathrm{w} / \mathrm{w}$ ( $c f$. Fig. $2 \mathrm{~b}$ and $3 \mathrm{~b}$, respectively), that show a markedly larger response in the IL boundary layer composition and extent into the bulk with applied potentials change at the gold electrode interface. The friction coefficient was also somewhat larger for $20 \% \mathrm{w} / \mathrm{w}$ than $5 \% \mathrm{w} / \mathrm{w}$, especially towards positive potentials. Along with the NR results this suggests that the adsorption behaviour of the IL on the gold electrode is quite different. Based on the NR results for $5 \% \mathrm{w} / \mathrm{w}$, the IL adsorption and friction is likely to be influenced by the specific interaction of the more lubricous cation with the gold electrode surface. However, for some other solvents, the integration of solvent molecules has been also shown to increase the strength of IL boundary layers, ${ }^{37}$ and thus play a vital part in the improvement of lubricity. ${ }^{38}$

By replotting the friction coefficient as a percentage of the friction coefficient at OCP $(\Delta \mu)$, we find that over a electrochemical range $+0.25 \mathrm{~V}$ to $-1 \mathrm{~V}$, the variance of the friction coefficient was $\Delta \mu=+3 \%$ to $-11 \%$ ( $c f$. Fig. $5 \mathrm{C}$ inset) for $5 \% \mathrm{w} / \mathrm{w}$ of $\left[\mathrm{P}_{6,6,6,14}\right][\mathrm{BMB}](\sim 0.67 \mathrm{~mol} \%)$. This electroresponse is significant compared to that reported for another phosphoniumbased IL on an $\mathrm{Au}(111)$ electrode in hexadecane at similar sliding velocity, that displayed no frictional electroresponsivity at a similar concentration $(1 \mathrm{~mol} \%) .{ }^{35}$ Such enhanced responsivity here is likely to be helped by the lower viscosity and higher dielectric constant of PC compared to hexadecane, making it a better charge carrying medium. Upon increasing the concentration to $20 \% \mathrm{w} / \mathrm{w}$, this variance in the friction coefficient increased to $\Delta \mu=+3$ to $-27 \%$.

\subsection{Electrochemical impedance spectroscopy}

EIS measures the differential interfacial capacitance, which is mainly determined by the charge distribution in the first few layers adjacent to the electrode, and is sensitive to
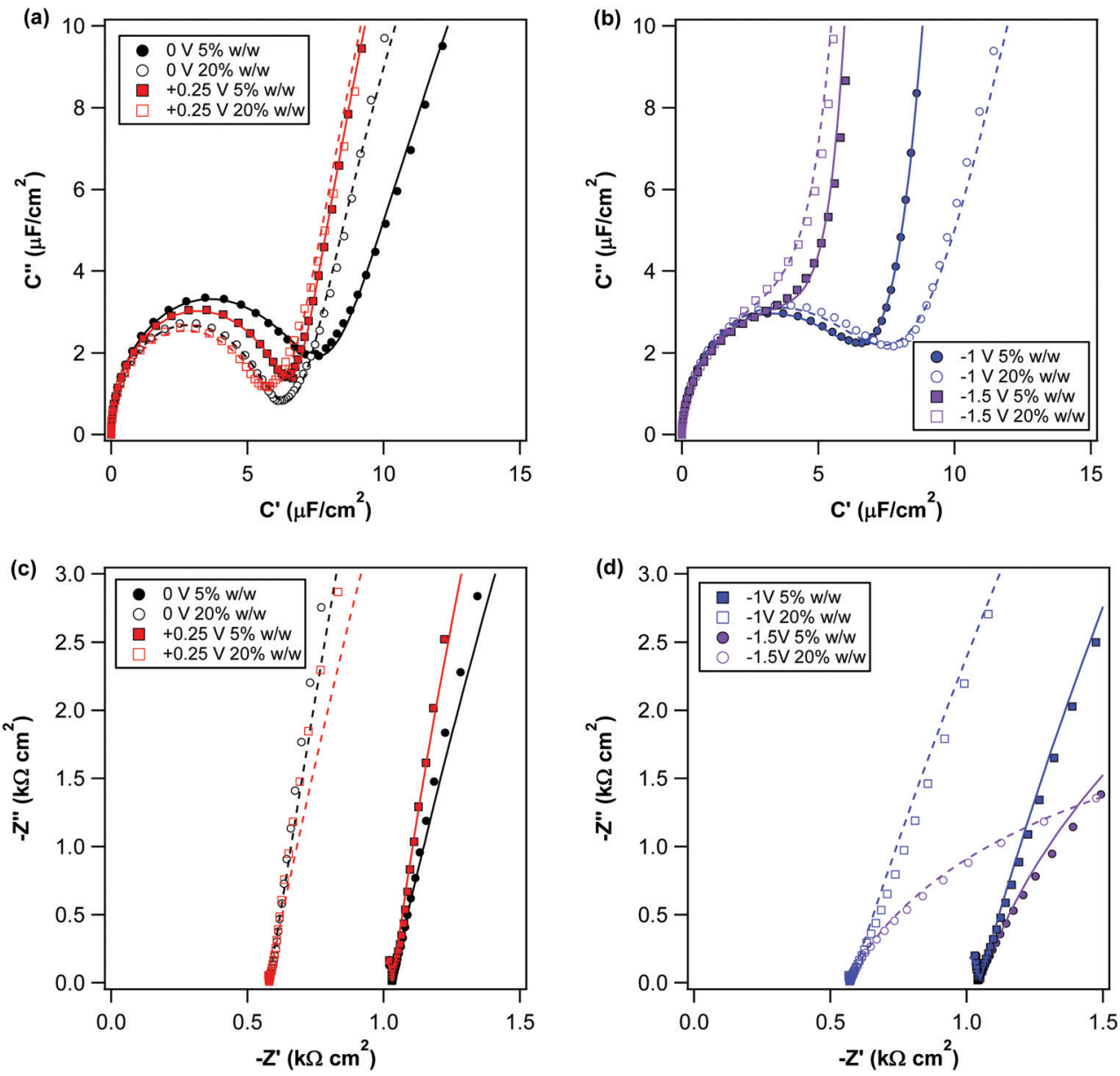

Fig. 6 Electrochemical impedance spectroscopy ( $a$ and b) complex capacitance plane and (c and d) Nyquist plots for 5 (filled symbols) and $20 \%$ w/w (open symbols) solutions of $\left[\mathrm{P}_{6,6,6,14}\right][\mathrm{BMB}]$ in $\mathrm{PC}$ for different applied potentials in the anodic $(a$ and $c$ ) and cathodic (b and d) regimes. The symbols represent experimental data, whilst the lines represent corresponding theoretical fits using an equivalent circuit mode (cf. ESI, $\dagger$ Fig. S3). $C^{\prime}$ and $C^{\prime \prime}$ are the real and imaginary parts of the capacitance, whilst $Z^{\prime}$ and $Z^{\prime \prime}$ are the real and imaginary parts of the impedance, respectively. 
conformational changes of the molecular ions in these layers. ${ }^{39}$ Fig. 6a and b show that at all direct current (DC) potentials studied, a clear semicircle representing a capacitive process is present. This "fast" capacitive process is typical in ILs and is related to all interfacial charge redistributions that do not require an activation energy. ${ }^{39-41}$ In some other cases, it is also possible to observe a second slow capacitive process related to charge redistributions in the innermost layer. ${ }^{42}$ This second semicircle is not observed in the $\left[\mathrm{P}_{6,6,6,14}\right][\mathrm{BMB}]-\mathrm{PC}$ solutions studied here. However, all the previously mentioned studies were conducted in neat ILs, whereas here we have studied mixtures with a low viscosity solvent (PC; $\eta=2.5 \mathrm{cP}$ at $25{ }^{\circ} \mathrm{C}$ ). Hence, we anticipate faster charge transfer processes to be present.

As discussed in Section 3.1, SLD profiles obtained from bestfits to the NR data reveal that the IL ions form a distinct interfacial region. The ionic composition of which is readily modified with applied potential. Although EIS measurements have not been performed on the pure IL, previous NR measurements for the same IL, in its pure form and when dispersed in another polar solvent (acetone), have similarly shown the presence of the solvent facilitates a greater redistribution of the ions when exposed to an electric field. ${ }^{24}$ Thus, the first and only capacitive process can be hypothesised to encompass all interfacial charge redistributions. Nyquist plots for the same data, plotted in Fig. $6 \mathrm{c}$ and d, show that the electrical impedance at any given frequency decreases with the IL bulk concentration, but is not significantly affected by the surface potential. The impedance over the high frequency range also decreases with increasing IL bulk concentration, indicating a greater adsorption of ions at the interface, which is concurrent with the model fits to the NR measurements and previous EIS measurements in IL solutions. ${ }^{22}$ A notable increase in the higher frequency range is also observed for the negative potential plot for $20 \% \mathrm{w} / \mathrm{w}$ and the positive potential for $5 \%$ $\mathrm{w} / \mathrm{w}$, consistent with the observed changes in the respective NR measurements above ( $c f$. Sections 3.1.1 and 3.1.3).

For a quantitative analysis of EIS measurements, complex impedance data are often fitted by equivalent circuits. Using an equivalent Randles circuit ( $c f$. ESI; $\dagger$ Fig. S3a), it is possible to estimate the differential capacitance using the Brug equation ${ }^{43}$ ( $c f$. ESI; $\dagger$ Fig. S3b). For the $20 \% \mathrm{w} / \mathrm{w}$ solution, a broad maxima in the capacitance between -1.5 to $-1 \mathrm{~V}$ is observed. Similar behaviour has been previously reported for another (pure) IL on gold and attributed to changes in the orientation of the IL cations in the innermost layers. ${ }^{42}$ Likewise, this potential range is found to correlate well with the cation-rich interface suggested the NR model fits for the $20 \% \mathrm{w} / \mathrm{w}$ IL solution at $-1.5 \mathrm{~V}$ ( $c f$. Fig. 4). In contrast, for the $5 \% \mathrm{w} / \mathrm{w}$ solution, a similar broad maximum is observed between -0.5 to $0.25 \mathrm{~V}$ ( $c f$. ESI; $\dagger$ Fig. S3b). Again, this feature is found to correlate well with the NR models presented (cf. Fig. 2b), which suggest a more significant rearrangement of the IL inner layer upon applying the positive potential $(+0.25 \mathrm{~V})$. Furthermore, an increase in capacitance is also consistent with a higher concentration of the anions near the electrode surface, as revealed by $\mathrm{NR}$, as the smaller size of the anions ( $c f$. Table 1) facilitates a larger number ions per area.

\section{Summary and conclusions}

The interfacial structuring and nanotribology of IL boundary layers at a gold electrode interface have been studied as a function of the bulk IL concentration and applied surface potential. NR data suggest that the IL forms a much thicker boundary layer than observed for similar bulk IL concentrations in acetone. ${ }^{24}$ For all IL concentrations a clear electroresponsive behaviour was observed. Both the composition and extent of the IL boundary layers showed a strong dependence on the bulk IL concentration at the different potential conditions. In particular, at the lowest IL concentration a specific interaction between the cation and gold was evident, which limited the electro-tunability of the layers, whilst for the higher IL concentrations distinct changes in the boundary layer and thickness were observed.

Clear tribotronic behaviour was also observed in complementary AFM nanotribology measurements on a gold electrode, whereby the friction coefficient $\mu$ reduced for larger biases of negative potentials and increased towards positive potentials. Together with the NR measurements, these results reveal a higher concentration of the phosphonium cation at negative potentials leads to the formation of a more compact boundary layer that leads to a significant reduction in the friction of an AFM tip against a gold surface compared to that of the pure solvent, PC. Meanwhile a higher concentration of anions and more diffuse interfacial structuring is shown to increase friction towards positive potentials, suggesting a lower load carrying capacity.

To provide further insight into the electroresponsivity of the IL layers, complementary EIS measurements were also performed for the lowest and highest IL bulk concentration at corresponding surface potentials. For all potentials, a fast capacitive process, attributed to charge redistributions, was observed and no evidence of slow capacitive processes detected, reflecting a large mobility of the IL ions. Consistent with the NR results presented, with increasing IL concentration the charge transfer resistance at the interface increased, indicating a greater concentration of ions in the boundary layers. Furthermore, larger changes in the impedance at higher frequencies also pointed towards greater structural changes in the IL layers for a positive bias at the lower concentration and a negative bias for the larger concentration. Such observations thus provide further evidence for a notably different surface interaction between the IL ions and the gold electrode depending on the bulk IL concentration.

Together the results clearly demonstrate the bulk IL concentration to be a critical parameter in IL tribotronic systems and point towards a minimum IL concentration being required in order to maximise electroresponsivity and friction control. More specifically it is proposed that in the case of the phosphonium-based IL studied here, specific ion-surface interactions between the cation and gold limited the tunability of the IL boundary layers with electric potential.

\section{Conflicts of interest}

There are no conflicts to declare. 


\section{Acknowledgements}

We acknowledge the Knut and Alice Wallenberg Foundation (Project No. KAW2012.0078), the Swedish Research Council (Project No. 2014-4694) and the Swedish Foundation for Strategic Research (Project No. EM16-0013) for their financial support. We gratefully acknowledge the Institut Laue-Langevin (ILL), France and ISIS Neutron and Muon Source, UK for access to neutron beamtime on the reflectometers SuperADAM (experiment no. CRG-2420 and 1-04-123) and INTER (experiment no. RB1810114; 10.5286/ISIS.E.RB1810114). GP would also like to thank Matthew Fielden for his help with the atomic force microscopy (AFM) measurements at the Nanofabrication facility at the Albanova University Center, Stockholm, Sweden. The authors also would like to thank Dr Benedikt Huber and Dr Marcel Druschler from rhd instruments $\mathrm{GmbH} \& \mathrm{Co}$. KG, Germany for their help with the EIS measurements. Dr Manishkumar Shimpi (Luleå University of Technology) is gratefully acknowledged for the synthesis of the IL.

\section{References}

1 M. Radiom, Curr. Opin. Colloid Interface Sci., 2019, 39, 148-161. 2 S. Glavatskih and E. Höglund, Tribol. Int., 2008, 41, 934-939.

3 J. Sweeney, F. Hausen, R. Hayes, G. B. Webber and F. Endres, et al., Phys. Rev. Lett., 2012, 109, 155502.

4 H. Li, R. J. Wood, M. W. Rutland and R. Atkin, Chem. Commun., 2014, 50, 4368-4370.

5 H. Li, M. W. Rutland and R. Atkin, Phys. Chem. Chem. Phys., 2013, 15, 14616-14623.

6 N. Hjalmarsson, D. Wallinder, S. Glavatskih, R. Atkin and T. Aastrup, et al., Nanoscale, 2015, 7, 16039-16045.

7 H. Li, M. W. Rutland, M. Watanabe and R. Atkin, Faraday Discuss., 2017, 199, 311-322.

8 J. Krim, Front. Mech. Eng., 2019, 5.

9 S. Watanabe, T. Koyama, Y. Kondo, K. Miyake and M. Nakano, et al., in Surfactants in Tribology, ed. K. L. M. G. Biresaw, CRC Press, 2015, vol. 4, ch. 9.

10 F. U. Shah, S. Glavatskih and O. N. Antzutkin, Tribol. Lett., 2013, 51, 281-301.

11 F. U. Shah, S. Glavatskih, D. R. MacFarlane, A. Somers and M. Forsyth, et al., Phys. Chem. Chem. Phys., 2011, 13, 12865-12873.

12 H. Li, A. E. Somers, P. C. Howlett, M. W. Rutland and M. Forsyth, et al., Phys. Chem. Chem. Phys., 2016, 18, 6541-6547.

13 H. Li, P. K. Cooper, A. E. Somers, M. W. Rutland and P. C. Howlett, et al., J. Phys. Chem. Lett., 2014, 5, 4095-4099.

14 H. Li, A. E. Somers, M. W. Rutland, P. C. Howlett and R. Atkin, ACS Sustainable Chem. Eng., 2016, 4, 5005-5012.

15 P. Rohlmann, B. Munavirov, I. Furó, O. Antzutkin and M. W. Rutland, et al., Front. Chem., 2019, 7.

16 N. Hjalmarsson, E. Bergendal, Y.-L. Wang, B. Munavirov and D. Wallinder, et al., Langmuir, 2019, 35, 15692-15700.

17 M. Ploss, Y. Tian, S. Yoshikawa, R. Westbroek and J. Leckner, et al., Tribol. Lett., 2019, 68, 3.
18 S. Watanabe, G. Pilkington, A. Oleshkevych, P. P. Carrasco and M. Radiom, et al., Phys. Chem. Chem. Phys., 2020, 22, 8450-8460.

19 M. Mezger, R. Roth, H. Schröder, P. Reichert and D. Pontoni, et al., J. Chem. Phys., 2015, 142, 164707.

20 L. Kong, W. Huang and X. Wang, J. Phys. D: Appl. Phys., 2016, 49, 225301.

21 X. Yang, Y. Meng and Y. Tian, Wear, 2016, 360-361, 104-113.

22 X. Yang, Y. Meng and Y. Tian, Tribol. Lett., 2014, 56, 161-169.

23 S. W. Coles, A. M. Smith, M. V. Fedorov, F. Hausen and S. Perkin, Faraday Discuss., 2018, 206, 427-442.

24 G. A. Pilkington, K. Harris, E. Bergendal, A. B. Reddy and G. K. Palsson, et al., J. Chem. Phys., 2018, 148, 193806.

25 Y. Lauw, M. D. Horne, T. Rodopoulos, V. Lockett and B. Akgun, et al., Langmuir, 2012, 28, 7374-7381.

26 Y.-L. Wang, M. Golets, B. Li, S. Sarman and A. Laaksonen, ACS Appl. Mater. Interfaces, 2017, 9, 4976-4987.

27 M. Björck and G. Andersson, J. Appl. Crystallogr., 2007, 40, 1174-1178.

28 A. Feiler, P. Attard and I. Larson, Rev. Sci. Instrum., 2000, 71, 2746-2750.

29 G. Bogdanovic, A. Meurk and M. W. Rutland, Colloids Surf., B, 2000, 19, 397-405.

30 R. Álvarez-Asencio, E. Thormann and M. W. Rutland, Rev. Sci. Instrum., 2013, 84, 096102.

31 T. Pettersson, N. Nordgren, M. W. Rutland and A. Feiler, Rev. Sci. Instrum., 2007, 78, 093702.

32 M. Radiom, P. Pedraz, G. Pilkington, P. Rohlmann and S. Glavatskih, et al., Colloids Interfaces, 2018, 2, 60.

33 P. K. Cooper, H. Li, N. R. Yepuri, A. Nelson and G. B. Webber, et al., J. Phys. Chem. C, 2018, 122, 24077-24084.

34 H. Li, R. J. Wood, F. Endres and R. Atkin, J. Phys.: Condens. Matter, 2014, 26, 284115.

35 P. K. Cooper, H. Li, M. W. Rutland, G. B. Webber and R. Atkin, Phys. Chem. Chem. Phys., 2016, 18, 23657-23662.

36 H. Li, M. W. Rutland, M. Watanabe and R. Atkin, Faraday Discuss., 2017, 199, 311-322.

37 M. Jitvisate and J. R. T. Seddon, J. Phys. Chem. C, 2017, 121, 18593-18597.

38 S. Cowie, P. K. Cooper, R. Atkin and H. Li, J. Phys. Chem. C, 2017, 121, 28348-28353.

39 B. Roling, M. Drüschler and B. Huber, Faraday Discuss., 2012, 154, 303-311.

40 J. Wallauer, M. Drüschler, B. Huber and B. Roling, Z. Naturforsch., B: J. Chem. Sci., 2013, 68, 1143.

41 R. Atkin, N. Borisenko, M. Drüschler, F. Endres and R. Hayes, et al., J. Mol. Liq., 2014, 192, 44-54.

42 R. Atkin, N. Borisenko, M. Drüschler, S. Z. El Abedin and F. Endres, et al., Phys. Chem. Chem. Phys., 2011, 13, 6849-6857.

43 G. J. Brug, A. L. G. van den Eeden, M. Sluyters-Rehbach and J. H. Sluyters, J. Electroanal. Chem. Interfacial Electrochem., 1984, 176, 275-295. 